\title{
Magnetic Field Measurements of LHC Inner Triplet Quadrupoles Fabricated at Fermilab
}

\author{
G.V. Velev, R. Bossert, R. Carcagno, J. DiMarco, S. Feher, V.V. Kashikhin, J. Kerby, M. Lamm, D. \\ Orris, P. Schlabach, J. Strait, C. Sylvester, M. Tartaglia, J.C. Tompkins, A.V. Zlobin
}

\begin{abstract}
Fermilab, as part of the US-LHC Accelerator Project, is producing superconducting low-beta quadrupole magnets for the Large Hadron Collider (LHC). These $5.5 \mathrm{~m}$ long magnets are designed to operate in superfluid helium at $1.9 \mathrm{~K}$ with a nominal gradient of $205 \mathrm{~T} / \mathrm{m}$ in the $70 \mathrm{~mm}$ bore. Two quadrupoles separated by a dipole orbit corrector in a single cryogenic assembly comprise the Q2 optical elements of the final focus triplets in the LHC interaction regions. The field quality of the quadrupoles is measured at room temperature during construction of the cold masses as well as during cold testing of the cryogenic assembly. We summarize data from the series measurements of the magnets and discuss various topics of interest.
\end{abstract}

Index Terms - Magnetic Field Measurements, Superconducting accelerator magnets

\section{INTRODUCTION}

$\mathrm{P}$ RODUCTION of the Large Hadron Collider (LHC) low- $\beta$ focusing quadrupoles at Fermilab is approaching its final stage. These magnets, arranged in a final focusing triplet Q1, Q2a, Q2b, Q3, (as shown in Fig. 1) are designed to perform at the LHC high luminosity regime $\left(10^{34} \mathrm{~cm}^{-2} \mathrm{~s}^{-1}\right)$ [1]. They have to provide a maximum operating gradient of $215 \mathrm{~T} / \mathrm{m}$ at $1.9 \mathrm{~K}$ over a relatively large aperture of $70 \mathrm{~mm}$ coil bore due to a large variation of the beam $\beta$-function in the interaction regions. Two different laboratories provided the cold masses for the low- $\beta$ quadrupoles. The first type, MQXA [2], was developed at KEK and produced by Toshiba in Japan. They were tested at $1.9 \mathrm{~K}$ at KEK, and supplied to Fermilab for final assembly into LQXA (Q1) and LQXC (Q3) cryogenic elements. The second type, MQXB (Q2a, Q2b) [3]-[5], was developed and produced by Fermilab: twenty MQXB have been built and, eighteen of them, together with the corresponding correctors, were selected to form nine LQXB cryogenic units, including one spare. To date, eight LQXB have been assembled and shipped to CERN.

In this paper, we present the results of magnetic measurements of the MQXBs. Room temperature

Manuscript received August 26, 2006. Work supported by the U.S. Department of Energy.
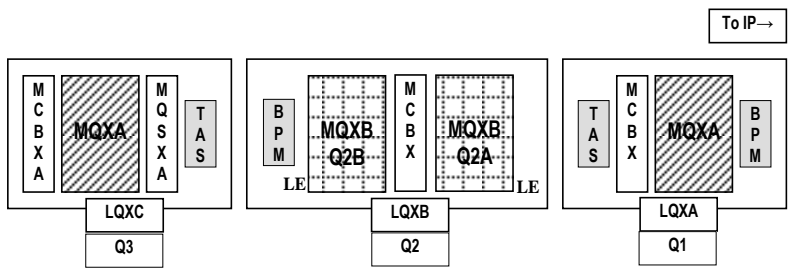

Fig. 1. Schematic view of the LHC focusing inner triplet system. The middle element in the triplet is the LQXB assembly and it consists of two oppositely positioned and electrically connected MQXB quadrupoles.

measurements were performed at the collared coil and yoked assembly stages as part of production quality control procedures. A full set of magnetic measurements were done during cold testing of the magnets. Results, including warmto-cold correlations, are summarized and discussed.

All results in this paper are expressed in terms of harmonic coefficients defined in a series expansion given by

$$
B_{y}+i B_{x}=B_{2} 10^{-4} \sum_{n=1}^{\infty}\left(b_{n}+i a_{n}\right)\left(\frac{x+i y}{r_{0}}\right)^{n-1}
$$

where $B_{x}$ and $B_{y}$ in (1) are the field components in the Cartesian coordinates, $b_{n}$ and $a_{n}$ are the 2n-pole normal and skew coefficients at the reference radius $r_{0}$ of $17 \mathrm{~mm}$, which was chosen for LHC $\left(b_{2}=10^{4}\right)$. Probe centering is done using the standard technique of zeroing the dipole component assuming that it is purely generated from a probe offset in the quadrupole field. The right-handed measurement coordinate system is defined from the point of view of the lead end where the positive $\mathrm{z}$-axis is along the magnet. In this coordinate system, an MQXB is a normal negatively powered quadrupole and as consequence, all field harmonics are reported for a negative main field.

\section{FIELD MEASUREMENTS}

Warm magnetic measurements were performed during the production process. Cold measurements were carried out at the Fermilab Magnet Test Facility. In both cases, the field harmonics were measured with a rotating coil system that is integrated with a probe translation system. A detailed description of the system can be found in [6].

All authors are with Fermilab, P.O. Box 500, Batavia, IL 60510, USA, (email: velev@fnal.gov; phone: 630-840-2203; fax: 630-840-8079). 
TABLE I

Average Room Temperature Field Harmonics in Straight Section

\begin{tabular}{|c|c|c|c|c|}
\hline \multirow{3}{*}{$\mathrm{n}$} & \multicolumn{2}{|c|}{ MQXB field harmonics } & \multicolumn{2}{|c|}{$\begin{array}{l}\text { Model program field } \\
\text { harmonics }\end{array}$} \\
\hline & $b_{n} \pm \Delta b_{n}$ & $a_{n} \pm \Delta a_{n}$ & $\mathrm{~b}_{\mathrm{n}} \pm \Delta \mathrm{b}_{\mathrm{n}}$ & $a_{n} \pm \Delta a_{n}$ \\
\hline & \multicolumn{4}{|c|}{ (Units at $17 \mathrm{~mm}$ ) } \\
\hline 3 & $-0.23 \pm 0.84$ & $-0.05 \pm 1.14$ & $0.01 \pm 0.50$ & $-0.69 \pm 0.56$ \\
\hline 4 & $0.02 \pm 0.12$ & $-0.05 \pm 0.53$ & $-0.18 \pm 0.33$ & $0.11 \pm 1.01$ \\
\hline 5 & $-0.02 \pm 0.15$ & $0.00 \pm 0.19$ & $-0.02 \pm 0.27$ & $-0.04 \pm 0.13$ \\
\hline 6 & $-1.25 \pm 0.30$ & $-0.04 \pm 0.07$ & $-1.53 \pm 0.37$ & $-0.05 \pm 0.20$ \\
\hline
\end{tabular}

\section{A. Field Measurements at Room Temperature}

During the process of production of each MQXB, two room temperature measurements were performed to monitor the initial magnet quality. The first measurement, a longitudinal scan of the collared coil, checked the integrity of the assembly. A similar scan followed the magnet yoking process. In both cases, the same tangential probe was utilized, and it was positioned in the longitudinal direction with a reproducibility of better than $\pm 0.5 \mathrm{~mm}$.

Table 1 shows the room temperature field harmonics averaged over $18 \mathrm{MQXB}$ collared coils (left columns). The right columns represent the averages from the last five $2 \mathrm{~m}$ long magnets from the model programs that preceded the full scale production [3], [7]. These five magnets were considered to have an accelerator quality field. The errors assigned to the means $\left(\Delta \mathrm{b}_{\mathrm{n}}\right.$ and $\left.\Delta \mathrm{a}_{\mathrm{n}}\right)$ correspond to the calculated RMS. Within the errors, there is good agreement between the field harmonics from both groups.

To decrease the absolute value of the normal dodecapole after the production of the first three magnets (MQXB01-03), a new coil-shimming pattern was adopted. The size of the shims was determined such that $b_{6}$ in the magnet body at the collision current $(11.3 \mathrm{kA}, 205 \mathrm{~T} / \mathrm{m})$ was slightly negative to compensate for the relatively large positive dodecapole in the lead end of the magnet.

Fig.2 shows the $b_{6}$ measured along the length of MQXB09 for the collared coil (circles) and the yoked magnet (squares).

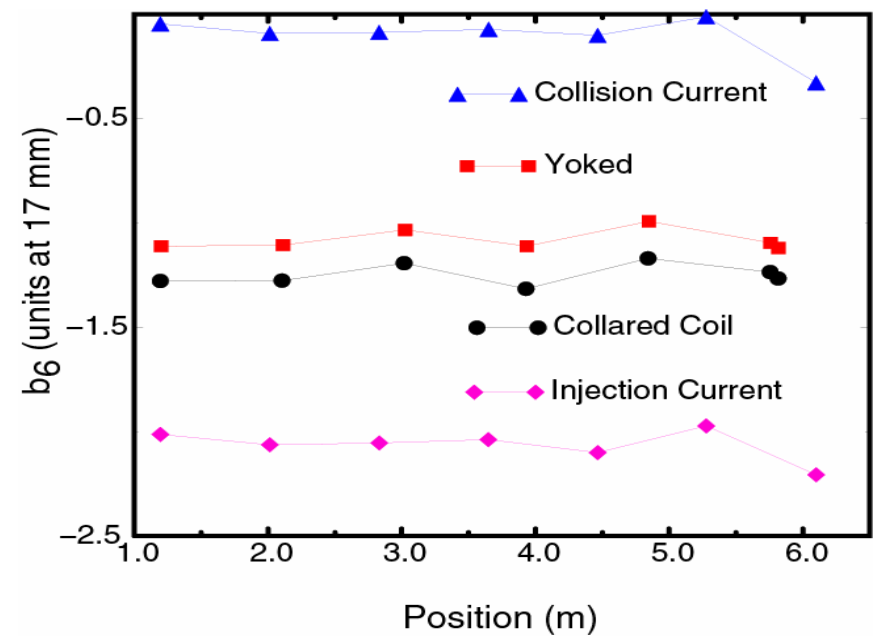

Fig. 2. Normal dodecapole profile along a typical MQXB measured at room temperature (circles and squares). The same profile measured cold at injection (diamonds) and collision (triangles) currents is also presented.
Additionally, for comparison, the data from the cold measurements (diamonds) at the injection current of $0.67 \mathrm{kA}$

TABLE II

AVERAGE FIELD HARMONICS AT INJECTION AND COLLISION CURRENT

\begin{tabular}{rcccc}
\hline \hline & \multicolumn{3}{c}{ MQXB harmonics at injection } & \multicolumn{2}{c}{$\begin{array}{c}\text { MQXB harmonics at } \\
\text { collision }\end{array}$} \\
$\mathrm{n}$ & $\mathrm{b}_{\mathrm{n}} \pm \Delta \mathrm{b}_{\mathrm{n}}$ & $\mathrm{a}_{\mathrm{n}} \pm \Delta \mathrm{a}_{\mathrm{n}}$ & $\mathrm{b}_{\mathrm{n}} \pm \Delta \mathrm{b}_{\mathrm{n}}$ & $\mathrm{a}_{\mathrm{n}} \pm \Delta \mathrm{a}_{\mathrm{n}}$ \\
& & (Units at $17 \mathrm{~mm})$ \\
\hline 3 & $0.15 \pm 0.63$ & $-0.14 \pm 1.15$ & $-0.04 \pm 0.59$ & $0.01 \pm 1.00$ \\
4 & $0.10 \pm 0.15$ & $-0.26 \pm 0.48$ & $0.13 \pm 0.13$ & $-0.22 \pm 0.40$ \\
5 & $-0.03 \pm 0.14$ & $-0.02 \pm 0.20$ & $0.00 \pm 0.17$ & $0.01 \pm 0.18$ \\
6 & $-1.58 \pm 0.39$ & $-0.07 \pm 0.06$ & $0.11 \pm 0.29$ & $-0.10 \pm 0.18$ \\
7 & $0.01 \pm 0.04$ & $-0.01 \pm 0.04$ & $-0.00 \pm 0.04$ & $-0.00 \pm 0.03$ \\
8 & $-0.02 \pm 0.01$ & $-0.03 \pm 0.04$ & $-0.01 \pm 0.01$ & $-0.00 \pm 0.03$ \\
9 & $-0.01 \pm 0.01$ & $0.00 \pm 0.01$ & $0.00 \pm 0.01$ & $0.00 \pm 0.01$ \\
10 & $0.05 \pm 0.02$ & $-0.00 \pm 0.01$ & $0.02 \pm 0.01$ & $-0.00 \pm 0.02$ \\
\hline \hline
\end{tabular}

(12.3 $\mathrm{T} / \mathrm{m})$ and at the collision current (triangles) are presented. One can observe how the geometrical value of $b_{6}$ changes during the production process (adding the magnet yoke), cooling the magnet (warm-to-cold change of the iron permeability and coil sizes) and magnet excitation (changes in the coil position due to electromagnetic forces) as well as the presence of a significant magnetization component of the field at injection.

\section{B. Cold Measurements}

The final set of magnetic measurements was performed at the LHC operational temperature (1.9 K superfluid helium) on all the MQXB cold masses assembled in cryogenic elements LQXB01-10. Two of the cold masses (MQXB04 and MQXB14), as parts of LQXB02 and LQXB07, respectively, were limited by quench performance [5], [8] and were not measured cold.

During LQXB measurements, the rotating coil probe was inserted from the lead end of the Q2B cold mass. (See Fig.1.) In this configuration, Q2A cold masses were accessed from the return end. To present data from the two magnets in the coordinate system defined previously, we transformed the Q2A measurements. We also account for the fact that as measured the Q2A was powered "backwards".

The average integral harmonics at injection current are presented in Table II (left two columns). The same analysis was performed at collision current and is presented in the right two columns. One can see that the average values are small and there are no built-in systematic multipoles due to the production process.

\section{LQXB Integral Harmonics}

The Q2 integral multipoles were calculated as an average of the individual integral harmonics of MQXB cold masses inside the corresponding LQXB cryogenic element. Results for the eight measured LQXB elements at the collision current are shown in Fig. 3. (The higher order harmonics are shown in the inset.) The open (filled) points represent normal (skew) multipoles. The error bands correspond to the $3 \sigma$ acceptance limits established for the LQXB magnets. One sees integral 


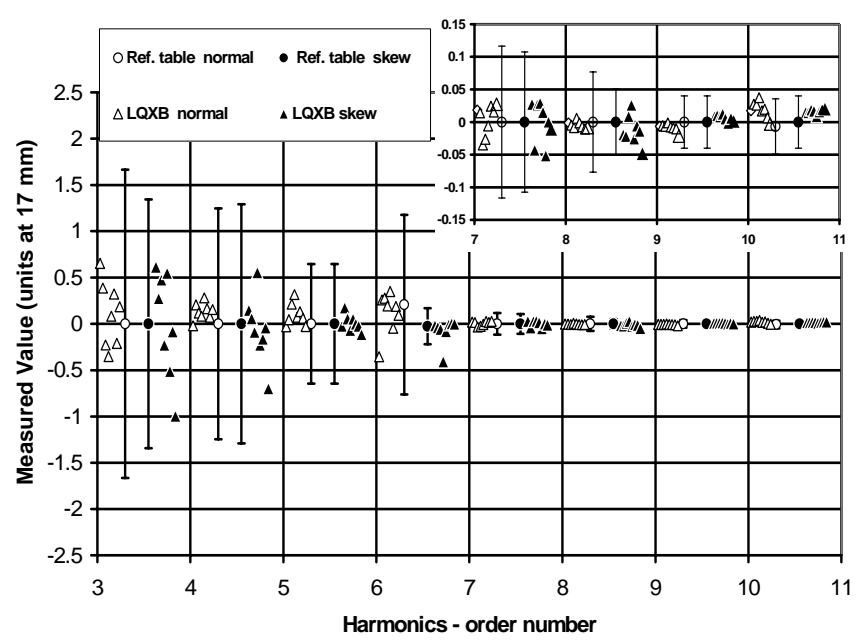

Fig. 3. Integral field harmonics (in units at $17 \mathrm{~mm}$ ) of the LQXB elements at 11.3 kA (LHC collision current). The inset shows the higher order harmonics. The points with error bars represent the acceptance limits.

field harmonics for the LQXB at LHC collision current are within the limits established during the model magnet program.

\section{LQXB Magnetic Length}

The magnetic length of accelerator magnets is an important part of their characterization. For a single quadrupole, it represents an effective dimension as if the magnet had a constant gradient along the body. For the LQXB element, we define the magnetic length as the sum of the individual MQXB quadrupole lengths:

$$
L_{L Q X B}=\sum_{Q 2 A, Q 2 B} \frac{\int B_{2} d z}{<B_{2}^{\text {magnet body }}>} .
$$

Fig. 4 shows the magnetic lengths of eight LQXB elements according to (2). The filled points correspond to the measured values for four different excitation currents. We observed a systematic change in the length from injection to collision current of $10 \mathrm{~mm}$, which we attributed to the stretching of the magnets under Lorentz forces. The open circle points with error bars are the average values for each LQXB element. The design value of $\boldsymbol{L}_{L Q X B}$ at room temperature warm is $11 \mathrm{~m}$ while the average cold one is $10.973 \pm 0.002 \mathrm{~m}$ at $1.9 \mathrm{~K}$. This change is about $0.24 \%$ of the magnet length at $1.9 \mathrm{~K}$ and corresponds to the thermal contraction of the stainless steel collars and the iron yoke - the dominant materials in the MQXB magnets.

\section{E. Warm to Cold Correlations}

The unallowed multipoles in the magnets are due to the inevitable imperfections in the coil geometry as a result of the manufacturing process. These imperfections change very little with magnet cooling. For this reason, one expects to observe a correlation between room temperature and cold magnetic measurements in most cases.

Figures 5 and 6 show the correlation plot for low and high order multipoles, respectively. The solid lines in the figures represent a one-to-one correlation. The data are very close to

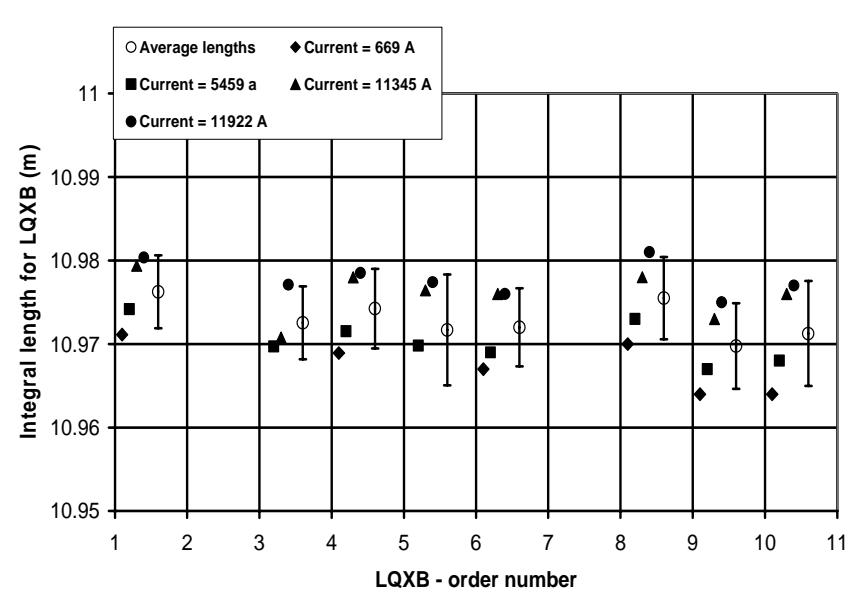

Fig. 4. Magnetic length of the LQXB elements at different excitation currents. The points with error bars represent the average values with their one sigma uncertainties.

this line except for $b_{6}, b_{10}$ and $a_{3}$. The allowed harmonics have an expected warm-to-cold shift (explained in Section II). We speculate that the noticeable difference in $\mathrm{a}_{3}$ in several of the magnets is due to changes in geometry during cooling.

\section{F. Dynamic Effects}

Dynamic effects in superconducting magnets play an important role in the operation of modern accelerators. This well-known phenomenon is usually associated with the decay and subsequent snapback of the allowed field components at

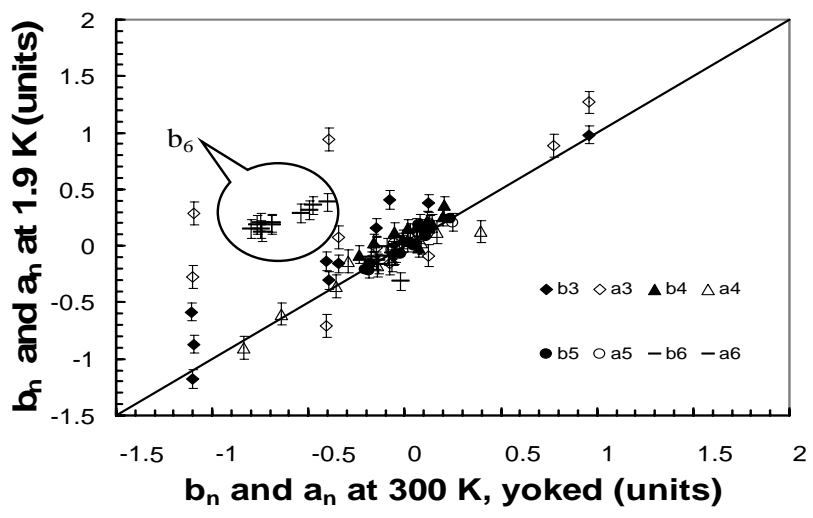

Fig. 5. Warm-to-cold correlation plot for the low order multipoles.

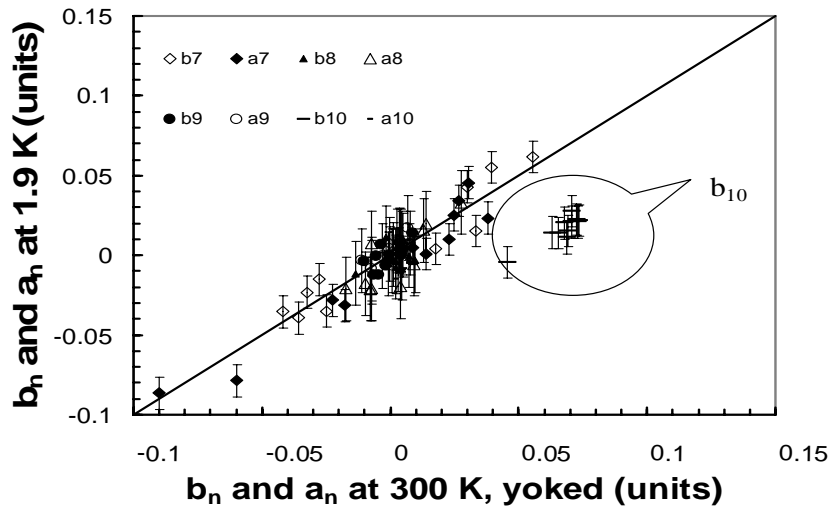

Fig. 6. Warm-to-cold correlation plot for the high order multipoles. 


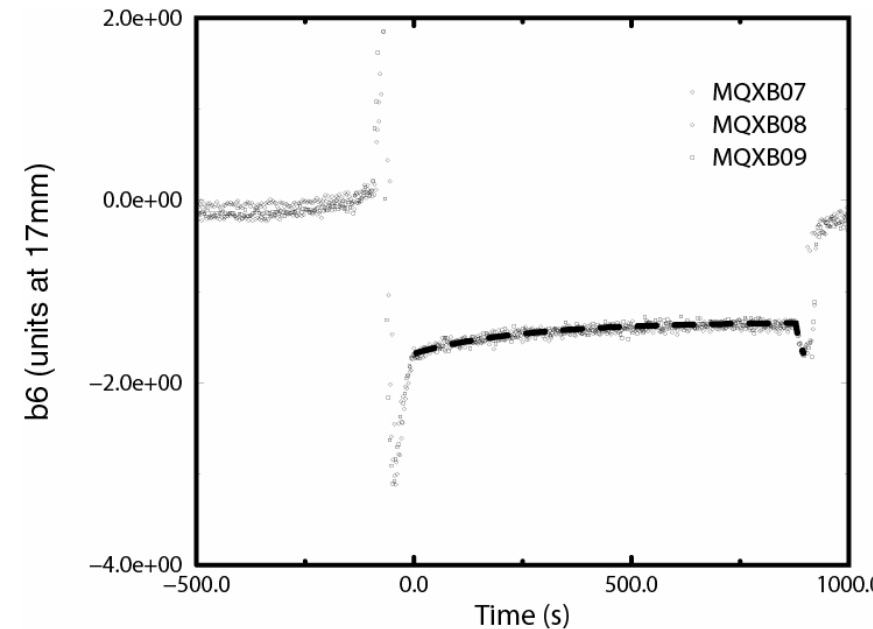

Fig. 7. Examples of the decay and snapback of the dodecapole component for a duration of injection of $\sim 900 \mathrm{~s}$ in three MQXB magnets. The dashed line is a fit of the decay and snapback according to [11], [12].

injection [10], [11]. To investigate these effects in the MQXB quadrupoles, we performed measurements with an accelerator current profile similar to the one used for the LHC arc dipole tests. The important characteristic of this profile is the duration of the injection plateau, which was set to $900 \mathrm{~s}$. In our measurements, we focused on the decay and snapback in normal dodecapole component, the first allowed multipole. Typical examples of the decay and snapback in MQXB quadrupoles are shown in Fig. 7. Table IV summarizes the decay amplitudes and snapback times for the MQXBs tested. The uncertainties, returned by the fit procedure, are approximately 0.05 units and $2 \mathrm{~s}$ for the decay amplitude and snapback time respectively. In this analysis, the decay and the snapback are parametrized with the logarithmic and gaussian functional forms typically used for correction of these effects in the Tevatron [11], [12]. The average decay amplitude is relatively small, approximately 0.4 units after $900 \mathrm{~s}$, followed by a snapback of $\sim 11 \mathrm{~s}$.

\section{SUMMARY}

The complete set of superconducting low-beta quadrupole cold masses for the LHC intersection regions has been produced. Warm and cold magnetic measurements showed field harmonics similar to those of model magnets produced during the latter stages of that program as well as those of the full-scale prototype [3], [7], [8]. Moreover, harmonics of the MQXBs measured at injection and collision currents at $1.9 \mathrm{~K}$ are small except for those with a magnetization component at injection. There is no evidence of systematic harmonics due to geometric imperfections resulting from the production process. LQXB integral harmonics at the collision current are within the acceptance limits established during the model magnet program. The magnet lengths of the LQXB elements at $1.9 \mathrm{~K}$ average $10.973 \mathrm{~m}$ with a variation at the $2 \times 10^{-3}$ level. Warm harmonics shows a good correlation with cold except for allowed harmonics, which shift systematically during cold down. In addition, dynamic effects were checked. The decay
TABLE IV

DECAY AMPLITUDE AND SNAPBACK TIME IN THE NORMAL DODECAPOLE

\begin{tabular}{ccc}
\hline \hline Cold mass & $\begin{array}{c}\text { Decay Amplitude* } \\
\text { (Units at 17 mm) }\end{array}$ & $\begin{array}{c}\text { Decay Time } \\
\text { (s) }\end{array}$ \\
\hline MQXB01 & 0.50 & 13.3 \\
MQXB02 & 0.35 & 8.9 \\
MQXB03 & 0.50 & 12.9 \\
MQXB05 & 0.30 & 9.8 \\
MQXB06 & 0.50 & 13.9 \\
MQXB08 & 0.26 & 8.1 \\
MQXB09 & 0.34 & 9.5 \\
MQXB10 & 0.35 & 9.8 \\
MQXB11 & 0.33 & 8.7 \\
MQXB12 & 0.55 & 15.3 \\
MQXB13 & 0.35 & 9.3 \\
MQXB17 & 0.54 & 15.6 \\
MQXB19 & 0.23 & 7.6 \\
Average & $0.39 \pm 0.11$ & $11.0 \pm 2.8$ \\
\hline \hline
\end{tabular}

* After a subtraction of the underlying hysteresis loop [12]

and snap-back after a $900 \mathrm{~s}$ injection time show an average decay of $b_{6}$ of $\sim 0.4$ units and average snapback time of $\sim 11 \mathrm{~s}$.

\section{REFERENCES}

[1] "The Large Hadron Collider conceptual design”, CERN AC/95-05, http://cdsweb.cern.ch.

[2] A.Yamamoto et al., "Production and measurement of the MQXA series of LHC low- $\beta$ insertion quadrupoles”, IEEE Trans. Appl. Supercond., vol. 15, 2005, pp. 1084-1089.

[3] N. Andreev et al., "Field quality in Fermilab-built models of high gradient quadrupole magnets for the LHC interaction region", IEEE Trans. Appl. Supercond., vol. 10, 2000, pp. 107-110.

[4] G. V. Velev et al., "Magnetic field measurements of the LHC inner triplet quadrupoles produced at Fermilab" in Proc. $9^{\text {th }}$ EPAC, Lucerne, 2004, pp. 1777-1779.

[5] M. J. Lamm et al., "Test results of Fermilab-built quadrupoles for the LHC interaction regions" in Proc. $10^{\text {th }}$ EPAC, Edinburgh, 2006, pp. 2637-2639.

[6] G.V. Velev et al., "Field quality measurements of the LQXB inner triplet quadrupoles for LHC”, IEEE Trans. Appl. Supercond., vol. 15, 2005, pp. 1102-1105.

[7] N. Andreev et al., "Quench performance of Fermilab high gradient quadrupole short models for LHC interaction regions" in Proc. $10^{\text {th }}$ PAC, New York, 1999, pp. 3197-3199.

[8] Bossert, et al., "Test results from the LQXB quadrupole production program at Fermilab for the LHC interaction regions", IEEE Trans. Appl. Supercond., vol. 14, 2004, pp. 187-190.

[9] N. Andreev et al., "Field quality in Fermilab-built models of quadrupole magnets for the LHC interaction region”, IEEE Trans. Appl. Supercond., vol. 11, 2001, pp. 1566-1569.

[10] D.A.Finley et al., "Time dependent chromaticity changes in the Tevatron“, in Proc. 1987 PAC., Washington, DC, 1987, pp 151-153.

[11] D.E. Johnson and D.A. Herrup "Compensation of time varying field in the Tevatron superconducting magnets", in Proc. 1989 PAC., Chicago, IL, 1989, pp. 521-523.

[12] G. Velev et al., "Measurements of sextupole decay and snapback in Tevatron dipole magnets", in Proc. ${ }^{\text {th }}$ EPAC, Lucerne, 2004, pp. 17801782. 